\title{
IMPLEMENTING A SUBCATEGORIZED Probabilistic Definite Clause Grammar for Vietnamese Sentence Parsing
}

\author{
Dang Tuan Nguyen, Kiet Van Nguyen, Tin Trung Pham \\ Faculty of Computer Science, University of Information Technology, \\ Vietnam National University - Ho Chi Minh City, \\ Ho Chi Minh City, Vietnam \\ \{ntdang, nvkiet, pttin\}@nlke-group.net
}

\begin{abstract}
In this paper, we introduce experiment results of a Vietnamese sentence parser which is built by using the Chomsky's subcategorization theory and PDCG (Probabilistic Definite Clause Grammar). The efficiency of this subcategorized PDCG parser has been proved by experiments, in which, we have built by hand a Treebank with 1000 syntactic structures of Vietnamese training sentences, and used different testing datasets to evaluate the results. As a result, the precisions, recalls and F-measures of these experiments are over $98 \%$.
\end{abstract}

\section{KEYWORDS}

Probabilistic Context-Free Grammar, Probabilistic Definite Clause Grammar, Parsing, Subcategorization

\section{INTRODUCTION}

The PCFG (Probabilistic Context-Free Grammar) [1], [2], [3], and [4] has been applied for developing some Vietnamese parsers as [5], [6], and [7]. All of these mentioned Vietnamese parsers use un-subcategorized PCFG.

In this research, we are interested in applying the Chomsky's subcategorization theory [8] and PDCG (Probabilistic Definite Clause Grammar) [9], [10], [11], and [12] to implement a subcategorized PDCG parser which allows analyzing effectively simple Vietnamese sentences. To implement this parser, we define our Vietnamese subcategorized PDCG grammar based on our set of sub-categorical and phrasal tags, and syntactic rules defined on these tags.

We also develop a Treebank for training this subcategorized PDCG parser. The Vietnamese sentences in our Treebank are syntactically analyzed and tagged by hand. 
International Journal on Natural Language Computing (IJNLC) Vol. 2, No.4, August 2013

\section{Sub-Categorical and Phrasal Tags}

\subsection{Definition of Subcategorical Tags}

\section{- Nominal Tags}

Nouns are divided into 9 sub-categories as presented in Table 1.

Table 1. Sub-categories of nouns

\begin{tabular}{|c|l|l|l|}
\hline No. & Nominal groups & Non-terminals & Adjectival tags \\
\hline 1 & Common nouns & n & N \\
\hline 2 & Abbreviation nouns & n_abbr & N_ABBR \\
\hline 3 & Currency nouns & n_currency & N_CURRENCY \\
\hline 4 & English nouns & n_eng & N_ENG \\
\hline 5 & Idiomatic nouns & n_idiom & N_IDIOM \\
\hline 6 & Proper nouns & n_prop & N_PROP \\
\hline 7 & Temporal nouns & n_time & N_TIME \\
\hline 8 & Title nouns & n_title & N_TITLE \\
\hline 9 & Unit nouns & n_unit & N_UNIT \\
\hline
\end{tabular}

\section{- Verbal tags}

Verbs are divided into 8 sub-categories as presented in Table 2.

Table 2. Sub-categories of verbs

\begin{tabular}{|c|l|l|l|}
\hline No. & Verbal groups & Non-terminals & Adjectival tags \\
\hline 1 & Ordinary verbs & v & V \\
\hline 2 & Passive verbs & v_bi & V_BI \\
\hline 3 & Motion verbs & v_di & V_DI \\
\hline 4 & Acquisition verbs & v_duoc & V_DUOC \\
\hline 5 & English verbs & v_eng & V_ENG \\
\hline 6 & Idiomatic verbs & v_idiom & V_IDIOM \\
\hline 7 & Là (to be) & v_la & V_LA \\
\hline 8 & Modal verbs & v_modal & V_MODAL \\
\hline
\end{tabular}

\section{- Adjectival tags}

Adjectives are divided into 10 sub-categories as presented in Table 3.

Table 3. Sub-categories of adjectives

\begin{tabular}{|c|l|l|l|}
\hline No. & \multicolumn{1}{|c|}{ Adjectival groups } & Non-terminals & Adjectival tags \\
\hline 1 & Qualitative adjective & adj & ADJ \\
\hline 2 & English adjective & adj_eng & ADJ_NUM \\
\hline 3 & Idiomatic adjective & adj_idiom & ADJ_PERCENT \\
\hline 4 & Measurement adjective & adj_measure & ADJ_QUANT \\
\hline 5 & Numeric adjective & adj_num & ADJ_NUM \\
\hline 6 & Ordinal adjective & adj_order & ADJ_ORDER \\
\hline
\end{tabular}


International Journal on Natural Language Computing (IJNLC) Vol. 2, No.4, August 2013

\begin{tabular}{|c|l|l|l|}
\hline 7 & Percentage adjective & adj_percent & ADJ_PERCENT \\
\hline 8 & Quantitative adjective & adj_quant & ADJ_QUANT \\
\hline 9 & Year adjective & adj_year & ADJ_YEAR \\
\hline 10 & Definite adjective & adj_dem & ADJ_DEM \\
\hline
\end{tabular}

\section{- Adverbial tags}

Adverbs are divided into 7 sub-categories as presented in Table 4.

Table 4. Sub-categories of adverbs

\begin{tabular}{|c|l|l|l|}
\hline No. & Adverbial groups & Non-terminals & Adverbial tags \\
\hline 1 & Ordinary adverb & adv & ADV \\
\hline 2 & Estimative adverb & adv_est & ADV_EST \\
\hline 3 & Adverb of frequency & adv_freq & ADV_FREG \\
\hline 4 & Negative adverb & adv_neg & ADV_NEG \\
\hline 5 & Ordinary adverb & adv_order & ADV_ORDER \\
\hline 6 & Adverb of time & adv_tense & ADV_TENSE \\
\hline 7 & Special adverb & adv_sp & ADV_SP \\
\hline
\end{tabular}

\section{- Prepositional tags}

Prepositions are divided into 3 groups, and ungrouped 17 prepositions. See in Table 5.

Table 5. Groups of prepositions

\begin{tabular}{|c|l|l|l|}
\hline No. & Prepositional groups & Non-terminals & Prepositional tags \\
\hline 1 & Preposition of cause & prep_cause & PREP_CAUSE \\
\hline 2 & Preposition of direction & prep_direct & PREP_DIRECT \\
\hline 3 & Preposition of location & prep_location & PREP_location \\
\hline 4 & Bằng & prep_bang & PREP_BANG \\
\hline 5 & Cho & prep_cho & PREP_CHO \\
\hline 6 & Của & prep_cua & PREP_CUA \\
\hline 7 & Cùng & prep_cung & PREP_CUNG \\
\hline 8 & Đề & prep_de & PREP_DE \\
\hline 9 & Khi & prep_khi & PREP_KHI \\
\hline 10 & Khỏi & prep_khoi & PREP_KHOI \\
\hline 11 & Không & prep_khong & PREP_KHONG \\
\hline 12 & Nếu & prep_neu & PREP_NEU \\
\hline 13 & Qua & prep_qua & PREP_QUA \\
\hline 14 & Sau & prep_sau & PREP_SAU \\
\hline 15 & Trong & prep_trong & PREP_TRONG \\
\hline 16 & Trước & prep_truoc & PREP_TRUOC \\
\hline 17 & Từ & prep_tu & PREP_TU \\
\hline 18 & Vào & prep_vao & PREP_VAO \\
\hline 19 & Về & prep_ve & PREP_VE \\
\hline 20 & Với & prep_voi & PREP_VOI \\
\hline
\end{tabular}


International Journal on Natural Language Computing (IJNLC) Vol. 2, No.4, August 2013

- Conjunctional tags

Table 6. Groups of prepositions

\begin{tabular}{|c|l|l|l|}
\hline No. & Conjunctional groups & Non-terminals & Conjunctional tags \\
\hline 1 & và, “-”, “,” & conj & CONJ \\
\hline
\end{tabular}

\section{- Special tags}

In Vietnamese, there are words that always precede a noun or an adjective to modify for the noun or adjective, e.g. "cố", "cựu", "phó", "siêu", "tân", ... We arrange these special words in a group.

Table 7. Groups of special words

\subsection{Phrasal tags}

\begin{tabular}{|c|l|l|l|}
\hline No. & Special groups & Non-terminals & Special tags \\
\hline 1 & Special words & sp_word & SP_WORD \\
\hline
\end{tabular}

- Verbal phrase tags

Verbal phrase are divides in to 5 groups as shown in Table 8.

Table 8. Groups of verbal phrases

\begin{tabular}{|c|l|l|l|}
\hline No. & \multicolumn{1}{|c|}{ Verbal phrase groups } & \multicolumn{1}{|c|}{ Non-terminals } & Verbal phrase tags \\
\hline 1 & Verbal phrase having a intransitive verb & $\mathrm{vp} 1$ & VP1 \\
\hline 2 & $\begin{array}{l}\text { Verbal phrase having a transitive verb and its } \\
\text { direct object }\end{array}$ & $\mathrm{vp} 2$ & $\mathrm{VP} 2$ \\
\hline 3 & $\begin{array}{l}\text { Verbal phrase having a transitive verb and its } \\
\text { indirect object }\end{array}$ & $\mathrm{vp} 3$ & $\mathrm{VP3}$ \\
\hline 4 & $\begin{array}{l}\text { Verbal phrase having a transitive verb and its } \\
\text { direct and indirect object }\end{array}$ & $\mathrm{vp} 4$ & $\mathrm{VP} 4$ \\
\hline 5 & General verbal phrase & $\mathrm{vp}$ & $\mathrm{VP}$ \\
\hline
\end{tabular}

- Nominal phrase tags

Noun phrases are divided into 8 groups, as presented in Table 9.

Table 9. Groups of nominal phrases

\begin{tabular}{|c|l|l|l|}
\hline No. & \multicolumn{1}{|c|}{ Nominal phrase groups } & \multicolumn{1}{|c|}{ Non-terminals } & Nominal phrase tags \\
\hline 1 & General noun phrase & np & NP \\
\hline 2 & $\begin{array}{l}\text { Noun phrases have two components linked } \\
\text { together by connected words or hyphen }\end{array}$ & np_conj & NP_CONJ \\
\hline 3 & Currency noun phrases & np_currency & NP_CURRENCY \\
\hline 4 & $\begin{array}{l}\text { Noun phrases contains one, or two, or three, } \\
\text { or four, or five noun(s) }\end{array}$ & $\begin{array}{l}\text { np_n } \\
\text { np_nn } \\
\text { np_nnn } \\
\text { np_nnnn } \\
\text { np_nnnnn }\end{array}$ & NP_N \\
& NP_NN \\
NP_NNN \\
NP_NNNN \\
NP_NNNNN \\
\hline
\end{tabular}


International Journal on Natural Language Computing (IJNLC) Vol. 2, No.4, August 2013

\begin{tabular}{|c|l|l|l|}
\hline 5 & $\begin{array}{l}\text { Noun phrases contain one, or two, or three } \\
\text { noun(s) that precede a preposition }\end{array}$ & $\begin{array}{l}\text { np_npp } \\
\text { np_nnpp } \\
\text { np_nnnpp }\end{array}$ & $\begin{array}{l}\text { NP_NPP } \\
\text { NP_NNPP } \\
\text { NP_NNNPP }\end{array}$ \\
\hline 6 & Noun phrases of pronoun & np_pn & NP_PN \\
\hline 7 & Noun phrases of proper name & np_prop & NP_PROP \\
\hline 8 & Noun phrases of time & np_time & NP_TIME \\
\hline
\end{tabular}

- Prepositional phrase tags

Prepositional phrases are divided into 15 groups based on preposition, as presented in Table 10.

Table 10. Groups of prepositional phrases

\begin{tabular}{|c|l|l|l|}
\hline No. & \multicolumn{1}{|c|}{ Prepositional groups } & Non-terminals & \multicolumn{1}{|c|}{$\begin{array}{c}\text { Prepositional } \\
\text { tags }\end{array}$} \\
\hline 1 & Prepositional phrases contain the preposition "bằng" & pp_bang & PP_BANG \\
\hline 2 & Prepositional phrases of cause & pp_cause & PP_CAUSE \\
\hline 3 & Prepositional phrases contain the preposition "cho" & pp_cho & PP_CHO \\
\hline 4 & Prepositional phrases contain the preposition "của" & pp_cua & PP_CUA \\
\hline 5 & Prepositional phrases contain the preposition "cùng" & pp_cung & PP_CUNG \\
\hline 6 & Prepositional phrases contain a preposition of direction & pp_direct & PP_DIRECT \\
\hline 7 & Prepositional phrases contain the preposition "khi" & pp_khi & PP_KHI \\
\hline 8 & Prepositional phrases contain the preposition "không" & pp_khong & PP_KHONG \\
\hline 9 & Prepositional phrases of location & pp_location & PP_LOCATION \\
\hline 10 & Prepositional phrases contain the preposition "qua" & pp_qua & PP_QUA \\
\hline 11 & Prepositional phrases contain the preposition "sau" & pp_sau & PP_SAU \\
\hline 12 & Prepositional phrases contain the preposition "trong" & pp_trong & PP_TRONG \\
\hline 13 & Prepositional phrases contain the preposition "trước" & pp_truoc & PP_TRUOC \\
\hline 14 & Prepositional phrases contain the preposition "vào" & pp_vao & PP_VAO \\
\hline 15 & Prepositional phrases contain the preposition "về" & pp_ve & PP_VE \\
\hline
\end{tabular}

\section{- Adjectival phrase tags}

Adjectival phrases are divided into 3 groups and presented in Table 11.

Table 11. Groups of adjectival phrases

\begin{tabular}{|c|l|l|l|}
\hline No. & \multicolumn{1}{|c|}{ Adjectival phrase groups } & Non-terminals & Adjectival phrase tags \\
\hline 1 & Adjectival phrase of quality & adjp & ADJP \\
\hline 2 & Adjectival phrase of number & adjp_num & ADJP_NUM \\
\hline 3 & Adjectival phrase of measurement & adjp_measure & ADJP_MEASURE \\
\hline
\end{tabular}

\section{Syntactic Rules of Phrases}

The probabilities of phrasal structure rules are calculated with 1000 Vietnamese training sentences in our TreeBank described in the experiments. 
International Journal on Natural Language Computing (IJNLC) Vol. 2, No.4, August 2013

\subsection{Nominal phrase}

- Nominal phrase of one noun

Nominal phrase NP_N is formed by a noun or/and an adjective modifying for noun.

Table 12. Syntactic rules of NP_N phrases

\begin{tabular}{|c|l|c|}
\hline No. & \multicolumn{1}{|c|}{ Rules } & Probabilities \\
\hline 1 & NP_N $\rightarrow$ ADJ_PRE N & 0.005714 \\
\hline 2 & NP_N $\rightarrow$ N & 0.809524 \\
\hline 3 & NP_N $\rightarrow$ N ADJP & 0.179048 \\
\hline 4 & NP_N $\rightarrow$ N ADJP_MEASURE & 0.001905 \\
\hline 5 & NP_N $\rightarrow$ N ADJP_NUM & 0.003810 \\
\hline
\end{tabular}

- Nominal phrase of two nouns

Nominal phrase NP_NN is formed by two nouns and without adjuncts.

Table 13. Syntactic rules of NP_NN phrases

\begin{tabular}{|c|c|c|}
\hline No. & Rules & Probabilities \\
\hline 1 & NP_NN $\rightarrow$ N N & 1.0 \\
\hline
\end{tabular}

- Nominal phrase of three nouns

Nominal phrase NP_NNN is formed by three nouns and without complements.

Table 14. Syntactic rules of NP_NNN phrases

\begin{tabular}{|c|c|c|}
\hline No. & Rules & Probabilities \\
\hline 1 & NP_NNN $\rightarrow$ N N N & 1.0 \\
\hline
\end{tabular}

- Noun phrase of four nouns

Nominal phrase NP_NNNN formed by four nouns and without complements.

Table 15. Syntactic rules of NP_NNNN phrases

\begin{tabular}{|c|c|c|}
\hline No. & Rules & Probabilities \\
\hline 1 & NP_NNNN $\rightarrow$ N N N N & 1.0 \\
\hline
\end{tabular}

- Nominal phrase of five nouns

Nominal phrase NP_NNNNN formed by five nouns and without complements.

Table 16. Syntactic rules of NP_NNNNN phrases

\begin{tabular}{|c|c|c|}
\hline No. & Rules & Probabilities \\
\hline 1 & NP_NNNNN $\rightarrow$ N N N N N & 1.0 \\
\hline
\end{tabular}


International Journal on Natural Language Computing (IJNLC) Vol. 2, No.4, August 2013

- Nominal phrase of proper nouns

Table 17. Syntactic rules of proper nouns

\begin{tabular}{|c|l|c|}
\hline No. & \multicolumn{1}{|c|}{ Rules } & Probabilities \\
\hline 1 & NP_PROP $\rightarrow$ N_PROP & 0.994715 \\
\hline 2 & NP_PROP $\rightarrow$ N_PROP N_PROP & 0.002114 \\
\hline 3 & NP_PROP $\rightarrow$ N_TITLE N_PROP & 0.003171 \\
\hline
\end{tabular}

- Nominal phrase of time

Table 18. Syntactic rules for nominal phrases of time

\begin{tabular}{|c|l|c|}
\hline No. & \multicolumn{1}{|c|}{ Rules } & Probabilities \\
\hline 1 & NP_TIME $\rightarrow$ ADJ_PRE N_TIME & 0.045455 \\
\hline 2 & NP_TIME $\rightarrow$ ADJ_PRE N_TIME ADJ_DEM & 0.022727 \\
\hline 3 & NP_TIME $\rightarrow$ ADJ_QUANT N_TIME & 0.022727 \\
\hline 4 & NP_TIME $\rightarrow$ ADJ_QUANT N_TIME ADJP_NUM & 0.022727 \\
\hline 5 & NP_TIME $\rightarrow$ ADJP_NUM N_TIME & 0.159091 \\
\hline 6 & NP_TIME $\rightarrow$ ADJP_NUM N_TIME ADJ_DEM & 0.022727 \\
\hline 7 & NP_TIME $\rightarrow$ ADJP_NUM N_TIME N & 0.022727 \\
\hline 8 & NP_TIME $\rightarrow$ N N_TIME & 0.022727 \\
\hline 9 & NP_TIME $\rightarrow$ N_TIME & 0.090909 \\
\hline 10 & NP_TIME $\rightarrow$ N_TIME ADJ & 0.045455 \\
\hline 11 & NP_TIME $\rightarrow$ N_TIME ADJ_DEM & 0.045455 \\
\hline 12 & NP_TIME $\rightarrow$ N_TIME ADJ_ORDER & 0.022727 \\
\hline 13 & NP_TIME $\rightarrow$ N_TIME ADJP_NUM & 0.045455 \\
\hline 14 & NP_TIME $\rightarrow$ N_TIME DATE & 0.022727 \\
\hline 15 & NP_TIME $\rightarrow$ N_TIME N & 0.318182 \\
\hline 16 & NP_TIME $\rightarrow$ N_TIME N N & 0.068182 \\
\hline
\end{tabular}

\section{- Nominal phrase of currency}

Table 19. Syntactic rules for nominal phrases of currency

\begin{tabular}{|c|c|c|}
\hline No. & Rules & Probabilities \\
\hline 1 & NP_CURRENCY $\rightarrow$ ADJP_NUM N_CURRENCY & 1.0 \\
\hline
\end{tabular}

- Nominal phrase containing conjunctions

Table 20. Syntactic rules of nominal phrases containing conjunctions

\begin{tabular}{|c|l|c|}
\hline No. & \multicolumn{1}{|c|}{ Rules } & Probabilities \\
\hline 1 & NP_CONJ $\rightarrow$ NP_N CONJ NP_N & 0.066667 \\
\hline 2 & NP_CONJ $\rightarrow$ NP_NN CONJ NP_NN & 0.038095 \\
\hline
\end{tabular}


International Journal on Natural Language Computing (IJNLC) Vol. 2, No.4, August 2013

\begin{tabular}{|c|l|c|}
\hline 3 & NP_CONJ $\rightarrow$ NP_PROP CONJ NP_N & 0.019048 \\
\hline 4 & NP_CONJ $\rightarrow$ NP_PROP CONJ NP_PROP & 0.819048 \\
\hline 5 & NP_CONJ $\rightarrow$ NP_PROP CONJ NP_PROP CONJ NP_PROP & 0.057143 \\
\hline
\end{tabular}

- Nominal phrase np_xpp is formed by np_n and pp_

Table 21. Syntactic rules of np_xpp

\begin{tabular}{|c|l|c|}
\hline No. & \multicolumn{1}{|c|}{ Rules } & Probabilities \\
\hline 1 & NP_NPP $\rightarrow$ NP_N PP_CUA & 0.688525 \\
\hline 2 & NP_NPP $\rightarrow$ NP_N PP_KHONG & 0.114754 \\
\hline 3 & NP_NPP $\rightarrow$ NP_N PP_LOCATION & 0.098361 \\
\hline 4 & NP_NPP $\rightarrow$ NP_N PP_TRONG & 0.032787 \\
\hline 5 & NP_NPP $\rightarrow$ NP_N PP_VE & 0.065574 \\
\hline
\end{tabular}

- Nominal phrase np_nnpp is formed by np_nn and pp_

Table 22. Syntactic rules of np_nnpp

\begin{tabular}{|c|l|c|}
\hline No. & \multicolumn{1}{|c|}{ Rules } & Probabilities \\
\hline 1 & NP_NNPP $\rightarrow$ NP_NN PP_CHO & 0.024390 \\
\hline 2 & NP_NNPP $\rightarrow$ NP_NN PP_CUA & 0.512195 \\
\hline 3 & NP_NNPP $\rightarrow$ NP_NN PP_LOCATION & 0.390244 \\
\hline 4 & NP_NNPP $\rightarrow$ NP_NN PP_QUA & 0.024390 \\
\hline 5 & NP_NNPP $\rightarrow$ NP_NN PP_TRONG & 0.048780 \\
\hline
\end{tabular}

- Nominal phrase np_nnnpp is formed by np_nnn and pp_

Table 23. Syntactic rules of np_nnnpp

\begin{tabular}{|c|l|c|}
\hline No. & \multicolumn{1}{|c|}{ Rules } & Probabilities \\
\hline 1 & NP_NNNPP $\rightarrow$ NP_NNN PP_CUA & 0.6 \\
\hline 2 & NP_NNNPP $\rightarrow$ NP_NNN PP_LOCATION & 0.3 \\
\hline 3 & NP_NNNPP $\rightarrow$ NP_NNN PP_TRONG & 0.1 \\
\hline
\end{tabular}

- Nominal phrase np_nnnnpp is formed by np_nnnn and pp_

Table 24. Syntactic rules of np_nnnnpp

\begin{tabular}{|c|c|c|}
\hline No. & Rules & Probabilities \\
\hline 1 & NP_NNNNPP $\rightarrow$ NP_NNNN PP_CUA. & 1.0 \\
\hline
\end{tabular}


International Journal on Natural Language Computing (IJNLC) Vol. 2, No.4, August 2013

- Nominal phrase

Table 25. Syntactic rules of general nominal phrases

\begin{tabular}{|c|c|c|}
\hline No. & Rules & Probabilities \\
\hline 1 & $\mathrm{NP} \rightarrow$ ADJ_PERCENT NP_N & $4.46 \mathrm{E}-04$ \\
\hline 2 & $\mathrm{NP} \rightarrow$ ADJ_PERCENT NP_NN & 8.93E-04 \\
\hline 3 & $\mathrm{NP} \rightarrow$ ADJ_QUANT NP_N & 0.003571 \\
\hline 4 & $\mathrm{NP} \rightarrow$ ADJ_QUANT NP_N PP_CUA & $8.93 \mathrm{E}-04$ \\
\hline 5 & $\mathrm{NP} \rightarrow$ ADJ_QUANT NP_N PP_LOCATION & $4.46 \mathrm{E}-04$ \\
\hline 6 & $\mathrm{NP} \rightarrow$ ADJ_QUANT NP_N PP_TRONG & $8.93 \mathrm{E}-04$ \\
\hline 7 & $\mathrm{NP} \rightarrow$ ADJ_QUANT NP_N PP_VOI & $8.93 \mathrm{E}-04$ \\
\hline 8 & $\mathrm{NP} \rightarrow$ ADJ_QUANT NP_NN & 0.002232 \\
\hline 9 & $\mathrm{NP} \rightarrow$ ADJ_QUANT NP_NN PP_CUA & $8.93 \mathrm{E}-04$ \\
\hline 10 & $\mathrm{NP} \rightarrow$ ADJ_QUANT NP_NN PP_TRONG & 0.001339 \\
\hline 11 & $\mathrm{NP} \rightarrow$ ADJ_QUANT NP_NNN & 0.001339 \\
\hline 12 & $\mathrm{NP} \rightarrow$ ADJ_QUANT NP_NNNN & $4.46 \mathrm{E}-04$ \\
\hline 13 & $\mathrm{NP} \rightarrow$ ADJP_MEASURE NP_N & $4.46 \mathrm{E}-04$ \\
\hline 14 & $\mathrm{NP} \rightarrow$ ADJP_MEASURE NP_NN & $4.46 \mathrm{E}-04$ \\
\hline 15 & $\mathrm{NP} \rightarrow$ ADJP_NUM NP_N & 0.012054 \\
\hline 16 & $\mathrm{NP} \rightarrow$ ADJP_NUM NP_N NP_TIME & $4.46 \mathrm{E}-04$ \\
\hline 17 & $\mathrm{NP} \rightarrow$ ADJP_NUM NP_N PP_CUA & $8.93 \mathrm{E}-04$ \\
\hline 18 & $\mathrm{NP} \rightarrow$ ADJP_NUM NP_N PP_TRONG & $4.46 \mathrm{E}-04$ \\
\hline 19 & $\mathrm{NP} \rightarrow$ ADJP_NUM NP_NN & 0.0125 \\
\hline 20 & $\mathrm{NP} \rightarrow$ ADJP_NUM NP_NN ADJP & $4.46 \mathrm{E}-04$ \\
\hline 21 & $\mathrm{NP} \rightarrow$ ADJP_NUM NP_NN PP_CUA & $8.93 \mathrm{E}-04$ \\
\hline 22 & NP $\rightarrow$ ADJP_NUM NP_NN PP_LOCATION & $4.46 \mathrm{E}-04$ \\
\hline 23 & $\mathrm{NP} \rightarrow$ ADJP_NUM NP_NNN & 0.002679 \\
\hline 24 & $\mathrm{NP} \rightarrow$ ADJP_NUM NP_NNNN & 0.001339 \\
\hline 25 & $\mathrm{NP} \rightarrow$ ADJP_NUM SP_WORD NP_N & $4.46 \mathrm{E}-04$ \\
\hline 26 & $\mathrm{NP} \rightarrow$ ADJP_NUM SP_WORD NP_NNN & $4.46 \mathrm{E}-04$ \\
\hline 27 & $\mathrm{NP} \rightarrow \mathrm{N}$ ADJ_QUANT NP_N & $4.46 \mathrm{E}-04$ \\
\hline 28 & $\mathrm{NP} \rightarrow \mathrm{N}$ ADJP_NUM NP_N & 0.002679 \\
\hline 29 & $\mathrm{NP} \rightarrow \mathrm{N}$ ADJP_NUM NP_NN & $4.46 \mathrm{E}-04$ \\
\hline 30 & $\mathrm{NP} \rightarrow \mathrm{N} \mathrm{NP} \_\mathrm{CONJ}$ & 0.008482 \\
\hline 31 & $\mathrm{NP} \rightarrow \mathrm{N}$ NP_CURRENCY & 0.001786 \\
\hline 32 & $\mathrm{NP} \rightarrow \mathrm{N}$ NP_CURRENCY ADJP_MEASURE & $4.46 \mathrm{E}-04$ \\
\hline 33 & $\mathrm{NP} \rightarrow \mathrm{N} \mathrm{NP} \_\mathrm{N}$ & $4.46 \mathrm{E}-04$ \\
\hline 34 & $\mathrm{NP} \rightarrow \mathrm{N}$ NP_PROP & $4.46 \mathrm{E}-04$ \\
\hline 35 & $\mathrm{NP} \rightarrow \mathrm{N} \mathrm{SP}$ _WORD NP_N & $4.46 \mathrm{E}-04$ \\
\hline 36 & $\mathrm{NP} \rightarrow \mathrm{N}$ SP_WORD NP_N ADJP & $4.46 \mathrm{E}-04$ \\
\hline 37 & $\mathrm{NP} \rightarrow \mathrm{N}$ SP_WORD NP_NN & 8.93E-04 \\
\hline
\end{tabular}


International Journal on Natural Language Computing (IJNLC) Vol. 2, No.4, August 2013

\begin{tabular}{|c|c|c|}
\hline 38 & $\mathrm{NP} \rightarrow$ NP_CONJ & 0.036161 \\
\hline 39 & $\mathrm{NP} \rightarrow$ NP_CONJ ADJP PP_LOCATION & $4.46 \mathrm{E}-04$ \\
\hline 40 & $\mathrm{NP} \rightarrow$ NP_CONJ PP_TRONG & $4.46 \mathrm{E}-04$ \\
\hline 41 & $\mathrm{NP} \rightarrow$ NP_CURRENCY & 0.003125 \\
\hline 42 & $\mathrm{NP} \rightarrow \mathrm{NP} \_\mathrm{N}$ & 0.165625 \\
\hline 43 & $\mathrm{NP} \rightarrow$ NP_N NP_PROP & 0.001339 \\
\hline 44 & $\mathrm{NP} \rightarrow \mathrm{NP} \_\mathrm{NN}$ & 0.215179 \\
\hline 45 & $\mathrm{NP} \rightarrow \mathrm{NP} \_$NN ADJP & 0.014732 \\
\hline 46 & $\mathrm{NP} \rightarrow$ NP_NN ADJP PP_CUA & 0.002232 \\
\hline 47 & $\mathrm{NP} \rightarrow$ NP_NN ADJP_NUM & $4.46 \mathrm{E}-04$ \\
\hline 48 & $\mathrm{NP} \rightarrow$ NP_NN ADJP_NUM NP_N & $4.46 \mathrm{E}-04$ \\
\hline 49 & $\mathrm{NP} \rightarrow \mathrm{NP} \_\mathrm{NN}$ NP_CONJ & 0.001339 \\
\hline 50 & NP $\rightarrow$ NP_NN NP_CURRENCY PP_CUA & $4.46 \mathrm{E}-04$ \\
\hline 51 & $\mathrm{NP} \rightarrow$ NP_NN NP_TIME & $4.46 \mathrm{E}-04$ \\
\hline 52 & NP $\rightarrow$ NP_NN NP_TIME PP_LOCATION & $4.46 \mathrm{E}-04$ \\
\hline 53 & $\mathrm{NP} \rightarrow$ NP_NN SP_WORD NP_NN & $4.46 \mathrm{E}-04$ \\
\hline 54 & $\mathrm{NP} \rightarrow \mathrm{NP} \_\mathrm{NNN}$ & 0.061161 \\
\hline 55 & $\mathrm{NP} \rightarrow$ NP_NNN ADJP & 0.001339 \\
\hline 56 & $\mathrm{NP} \rightarrow$ NP_NNN ADJP PP_CUA & $4.46 \mathrm{E}-04$ \\
\hline 57 & $\mathrm{NP} \rightarrow$ NP_NNN NP_TIME & $4.46 \mathrm{E}-04$ \\
\hline 58 & $\mathrm{NP} \rightarrow \mathrm{NP} \_\mathrm{NNNN}$ & 0.009821 \\
\hline 59 & $\mathrm{NP} \rightarrow \mathrm{NP} \_$NNNNN & 0.001339 \\
\hline 60 & $\mathrm{NP} \rightarrow \mathrm{NP} \_$NNNNPP & $8.93 \mathrm{E}-04$ \\
\hline 61 & $\mathrm{NP} \rightarrow \mathrm{NP} \_$NNNPP & 0.004464 \\
\hline 62 & $\mathrm{NP} \rightarrow \mathrm{NP} \_\mathrm{NNPP}$ & 0.017857 \\
\hline 63 & $\mathrm{NP} \rightarrow$ NP_NNPP NP_TIME & $4.46 \mathrm{E}-04$ \\
\hline 64 & $\mathrm{NP} \rightarrow$ NP_NPP & 0.026339 \\
\hline 65 & $\mathrm{NP} \rightarrow$ NP_NPP PP_LOCATION & $8.93 \mathrm{E}-04$ \\
\hline 66 & $\mathrm{NP} \rightarrow$ NP_PN & $4.46 \mathrm{E}-04$ \\
\hline 67 & $\mathrm{NP} \rightarrow$ NP_PROP & 0.331696 \\
\hline 68 & $\mathrm{NP} \rightarrow$ NP_PROP ADJP & $4.46 \mathrm{E}-04$ \\
\hline 69 & $\mathrm{NP} \rightarrow$ NP_PROP ADJP PP_LOCATION & $8.93 \mathrm{E}-04$ \\
\hline 70 & $\mathrm{NP} \rightarrow$ NP_PROP NP_TIME & $4.46 \mathrm{E}-04$ \\
\hline 71 & $\mathrm{NP} \rightarrow$ NP_PROP PP_CUA PP_LOCATION & $4.46 \mathrm{E}-04$ \\
\hline 72 & $\mathrm{NP} \rightarrow$ NP_PROP PP_TRUOC & $4.46 \mathrm{E}-04$ \\
\hline 73 & $\mathrm{NP} \rightarrow \mathrm{NP} \_$TIME & 0.011161 \\
\hline 74 & $\mathrm{NP} \rightarrow$ NP_TIME ADJP PP_LOCATION & $4.46 \mathrm{E}-04$ \\
\hline 75 & $\mathrm{NP} \rightarrow$ NP_TIME PP_CUA PP_LOCATION & $4.46 \mathrm{E}-04$ \\
\hline 76 & $\mathrm{NP} \rightarrow$ NP_TIME PP_LOCATION & $4.46 \mathrm{E}-04$ \\
\hline 77 & $\mathrm{NP} \rightarrow$ SP_WORD NP_N & 0.001786 \\
\hline 78 & $\mathrm{NP} \rightarrow$ SP_WORD NP_N PP_CUA & $4.46 \mathrm{E}-04$ \\
\hline
\end{tabular}


International Journal on Natural Language Computing (IJNLC) Vol. 2, No.4, August 2013

\begin{tabular}{|c|l|c|}
\hline 79 & NP $\rightarrow$ SP_WORD NP_NN & 0.013839 \\
\hline 80 & NP $\rightarrow$ SP_WORD NP_NN PP_LOCATION & $4.46 \mathrm{E}-04$ \\
\hline 81 & NP $\rightarrow$ SP_WORD NP_NNN & 0.001339 \\
\hline 82 & NP $\rightarrow$ SP_WORD NP_NNNN & $8.93 \mathrm{E}-04$ \\
\hline
\end{tabular}

\subsection{Verbal phrase}

- VP1: Verbal phrases only contain intransitive verb

Table 26. Syntactic rules of VP1

\begin{tabular}{|c|l|c|}
\hline No. & \multicolumn{1}{|c|}{ Rules } & Probabilities \\
\hline 1 & VP1 $\rightarrow$ ADV V & 0.040404 \\
\hline 2 & VP1 $\rightarrow$ ADV_NEG V & 0.020202 \\
\hline 3 & VP1 $\rightarrow$ ADV_TENSE V & 0.050505 \\
\hline 4 & VP1 $\rightarrow$ ADV_TENSE V ADV & 0.020202 \\
\hline 5 & VP1 $\rightarrow$ V & 0.737374 \\
\hline 6 & VP1 $\rightarrow$ V ADV & 0.121212 \\
\hline 7 & VP1 $\rightarrow$ V ADV_SP & 0.010101 \\
\hline
\end{tabular}

- VP2: Verbal phrases with a direct object of transitive verb

Table 27. Syntactic rules of VP2

\begin{tabular}{|c|l|c|}
\hline No. & \multicolumn{1}{|c|}{ Rules } & Probabilities \\
\hline 1 & VP2 $\rightarrow$ ADV V NP & 0.044444 \\
\hline 2 & VP2 $\rightarrow$ ADV V_LA NP & 0.001852 \\
\hline 3 & VP2 $\rightarrow$ ADV_FREQ V_LA NP & 0.001852 \\
\hline 4 & VP2 $\rightarrow$ ADV_NEG V NP & 0.012963 \\
\hline 5 & VP2 $\rightarrow$ ADV_NEG V_MODAL NP & 0.001852 \\
\hline 6 & VP2 $\rightarrow$ ADV_TENSE ADV V NP & 0.001852 \\
\hline 7 & VP2 $\rightarrow$ ADV_TENSE ADV_NEG V NP & 0.001852 \\
\hline 8 & VP2 $\rightarrow$ ADV_TENSE V ADV NP & 0.005556 \\
\hline 9 & VP2 $\rightarrow$ ADV_TENSE V NP & 0.068519 \\
\hline 10 & VP2 $\rightarrow$ V ADV NP & 0.044444 \\
\hline 11 & VP2 $\rightarrow$ V NP & 0.790741 \\
\hline 12 & VP2 $\rightarrow$ V NP ADV & 0.011111 \\
\hline 13 & VP2 $\rightarrow$ V_DUOC NP & 0.001852 \\
\hline 14 & VP2 $\rightarrow$ V_LA NP & 0.011111 \\
\hline
\end{tabular}


International Journal on Natural Language Computing (IJNLC) Vol. 2, No.4, August 2013

- VP3: Verbal phrases with an indirect object of transitive verb

Table 28. Syntactic rules of VP3

\begin{tabular}{|c|c|c|}
\hline No. & Rules & Probabilities \\
\hline 1 & VP3 $\rightarrow$ ADV V PREP_CAUSE NP & 0.009479 \\
\hline 2 & VP3 $\rightarrow$ ADV V PREP_TRUOC NP & 0.009479 \\
\hline 3 & VP3 $\rightarrow$ ADV V PREP_VE NP & 0.009479 \\
\hline 4 & VP3 $\rightarrow$ ADV V ADV PREP_CAUSE VP1 & 0.004739 \\
\hline 5 & VP3 $\rightarrow$ ADV_NEG ADV V PREP_LOCATION NP & 0.004739 \\
\hline 6 & VP3 $\rightarrow$ ADV_NEG V PREP_CHO NP & 0.004739 \\
\hline 7 & VP3 $\rightarrow$ ADV_NEG V PREP_TRONG NP & 0.004739 \\
\hline 8 & VP3 $\rightarrow$ ADV_NEG V PREP_VOI NP & 0.009479 \\
\hline 9 & VP3 $\rightarrow$ ADV_TENSE V PREP_LOCATION NP & 0.023697 \\
\hline 10 & VP3 $\rightarrow$ ADV_TENSE V PREP_TRONG NP & 0.014218 \\
\hline 11 & VP3 $\rightarrow$ ADV_TENSE V PREP_VE NP & 0.004739 \\
\hline 12 & VP3 $\rightarrow$ ADV_TENSE V PREP_VOI NP & 0.004739 \\
\hline 13 & VP3 $\rightarrow$ V ADV PREP_CAUSE NP & 0.014218 \\
\hline 14 & VP3 $\rightarrow$ V ADV PREP_CAUSE V_BI VP1 & 0.004739 \\
\hline 15 & VP3 $\rightarrow$ V ADV PREP_DE VP2 & 0.004739 \\
\hline 16 & VP3 $\rightarrow$ V ADV PREP_KHI V_BI VP3 & 0.004739 \\
\hline 17 & VP3 $\rightarrow$ V ADV PREP_KHI VP3 & 0.004739 \\
\hline 18 & VP3 $\rightarrow$ V ADV PREP_LOCATION NP & 0.014218 \\
\hline 19 & VP3 $\rightarrow$ V ADV PREP_QUA NP & 0.004739 \\
\hline 20 & VP3 $\rightarrow$ V ADV PREP_SAU NP & 0.004739 \\
\hline 21 & VP3 $\rightarrow$ V ADV PREP_TRONG NP & 0.004739 \\
\hline 22 & VP3 $\rightarrow$ V ADV PREP_TRUOC NP & 0.004739 \\
\hline 23 & VP3 $\rightarrow$ V ADV PREP_VE NP & 0.004739 \\
\hline 24 & VP3 $\rightarrow$ V ADV_SP PREP_VE NP & 0.004739 \\
\hline 25 & VP3 $\rightarrow$ V PREP_BANG NP & 0.014218 \\
\hline 26 & VP3 $\rightarrow$ V PREP_CAUSE NP & 0.113744 \\
\hline 27 & VP3 $\rightarrow$ V PREP_CAUSE V_BI VP1 & 0.004739 \\
\hline 28 & VP3 $\rightarrow$ V PREP_CAUSE V_BI VP2 & 0.004739 \\
\hline 29 & VP3 $\rightarrow$ V PREP_CAUSE VP1 & 0.018957 \\
\hline 30 & VP3 $\rightarrow$ V PREP_CAUSE VP2 & 0.033175 \\
\hline 31 & VP3 $\rightarrow$ V PREP_CHO NP & 0.023697 \\
\hline 32 & VP3 $\rightarrow$ V PREP_CUNG NP & 0.028436 \\
\hline 33 & VP3 $\rightarrow$ V PREP_DE VP1 & 0.014218 \\
\hline 34 & VP3 $\rightarrow$ V PREP_DE VP2 & 0.009479 \\
\hline 35 & VP3 $\rightarrow$ V PREP_DIRECT NP & 0.094787 \\
\hline 36 & VP3 $\rightarrow$ V PREP_KHI VP1 & 0.009479 \\
\hline 37 & VP3 $\rightarrow$ V PREP_KHOI NP & 0.004739 \\
\hline
\end{tabular}


International Journal on Natural Language Computing (IJNLC) Vol. 2, No.4, August 2013

\begin{tabular}{|c|l|c|}
\hline 38 & VP3 $\rightarrow$ V PREP_LOCATION NP & 0.156398 \\
\hline 39 & VP3 $\rightarrow$ V PREP_QUA NP & 0.009479 \\
\hline 40 & VP3 $\rightarrow$ V PREP_SAU NP & 0.014218 \\
\hline 41 & VP3 $\rightarrow$ V PREP_TRONG NP & 0.047393 \\
\hline 42 & VP3 $\rightarrow$ V PREP_TRUOC NP & 0.018957 \\
\hline 43 & VP3 $\rightarrow$ V PREP_TU NP & 0.004739 \\
\hline 44 & VP3 $\rightarrow$ V PREP_VE NP & 0.151659 \\
\hline 45 & VP3 $\rightarrow$ V PREP_VE VP2 & 0.004739 \\
\hline 46 & VP3 $\rightarrow$ V PREP_VOI NP & 0.009479 \\
\hline 47 & VP3 $\rightarrow$ V CONJ V ADV PREP_LOCATION NP & 0.004739 \\
\hline 48 & VP3 $\rightarrow$ V CONJ V PREP_VOI NP & 0.004739 \\
\hline 49 & VP3 $\rightarrow$ V_DI ADV PREP_TU NP & 0.004739 \\
\hline 50 & VP3 $\rightarrow$ V_DI PREP_DIRECT NP & 0.004739 \\
\hline 51 & VP3 $\rightarrow$ V_LA PREP_CAUSE NP & 0.009479 \\
\hline
\end{tabular}

- VP4: Verbal phrases with a direct object and an indirect object of transitive verb

Table 29. Syntactic rules of VP4

\begin{tabular}{|c|l|c|}
\hline No. & \multicolumn{1}{|c|}{ Rules } & Probabilities \\
\hline 1 & VP4 $\rightarrow$ ADV V NP PREP_DIRECT NP & 0.013245 \\
\hline 2 & VP4 $\rightarrow$ ADV_NEG V NP PREP_KHOI NP & 0.006623 \\
\hline 3 & VP4 $\rightarrow$ ADV_TENSE V ADV NP PREP_TU NP & 0.006623 \\
\hline 4 & VP4 $\rightarrow$ ADV_TENSE V NP PREP_CAUSE NP & 0.006623 \\
\hline 5 & VP4 $\rightarrow$ ADV_TENSE V NP PREP_CHO NP & 0.013245 \\
\hline 6 & VP4 $\rightarrow$ ADV_TENSE V NP PREP_CUNG NP & 0.006623 \\
\hline 7 & VP4 $\rightarrow$ ADV_TENSE V NP PREP_DIRECT NP & 0.052980 \\
\hline 8 & VP4 $\rightarrow$ V ADV NP PREP_DE VP3 & 0.006623 \\
\hline 9 & VP4 $\rightarrow$ V ADV NP PREP_CHO NP & 0.006623 \\
\hline 10 & VP4 $\rightarrow$ V ADV NP PREP_DIRECT NP & 0.013245 \\
\hline 11 & VP4 $\rightarrow$ V NP PREP_CAUSE ADV_NEG V_DUOC VP1 & 0.006623 \\
\hline 12 & VP4 $\rightarrow$ V NP PREP_CAUSE VP1 & 0.006623 \\
\hline 13 & VP4 $\rightarrow$ V NP PREP_CAUSE VP2 & 0.019868 \\
\hline 14 & VP4 $\rightarrow$ V NP PREP_DE VP1 & 0.006623 \\
\hline 15 & VP4 $\rightarrow$ V NP PREP_DE VP2 & 0.052980 \\
\hline 16 & VP4 $\rightarrow$ V NP PREP_DIRECT VP1 & 0.006623 \\
\hline 17 & VP4 $\rightarrow$ V NP PREP_KHI VP2 & 0.019868 \\
\hline 18 & VP4 $\rightarrow$ V NP PREP_BANG NP & 0.013245 \\
\hline 19 & VP4 $\rightarrow$ V NP PREP_CAUSE NP & 0.072848 \\
\hline 20 & VP4 $\rightarrow$ V NP PREP_CHO NP & 0.225166 \\
\hline 21 & VP4 $\rightarrow$ V NP PREP_CUNG NP & 0.006623 \\
\hline 22 & VP4 $\rightarrow$ V NP PREP_DIRECT ADV NP & 0.006623 \\
\hline 23 & VP4 $\rightarrow$ V NP PREP_DIRECT NP & 0.172185 \\
\hline & & \\
\hline
\end{tabular}


International Journal on Natural Language Computing (IJNLC) Vol. 2, No.4, August 2013

\begin{tabular}{|l|l|l|}
\hline 24 & VP4 $\rightarrow$ V NP PREP_KHOI NP & 0.019868 \\
\hline 25 & VP4 $\rightarrow$ V NP PREP_QUA NP & 0.006623 \\
\hline 26 & VP4 $\rightarrow$ V NP PREP_TRONG NP & 0.039735 \\
\hline 27 & VP4 $\rightarrow$ V NP PREP_TU NP & 0.019868 \\
\hline 28 & VP4 $\rightarrow$ V NP PREP_VE NP & 0.139073 \\
\hline 29 & VP4 $\rightarrow$ V NP PREP_CAUSE V VP2 & 0.006623 \\
\hline 30 & VP4 $\rightarrow$ V_BI NP PREP_CAUSE NP & 0.006623 \\
\hline 31 & VP4 $\rightarrow$ V_DUOC NP PREP_CHO VP1 & 0.006623 \\
\hline 32 & VP4 $\rightarrow$ V_LA NP PREP_CHO NP & 0.006623 \\
\hline
\end{tabular}

\section{- General verbal phrases}

Table 30. Syntactic rules of general verbal phrase

\begin{tabular}{|c|l|c|}
\hline No. & \multicolumn{1}{|c|}{ Rules } & Probabilities \\
\hline 1 & VP $\rightarrow$ ADV V VP1 & 0.008734 \\
\hline 2 & VP $\rightarrow$ ADV V VP2 & 0.021834 \\
\hline 3 & VP $\rightarrow$ ADV V_BI VP2 & 0.008734 \\
\hline 4 & VP $\rightarrow$ ADV V_BI VP3 & 0.004367 \\
\hline 5 & VP $\rightarrow$ ADV V_DI VP1 & 0.008734 \\
\hline 6 & VP $\rightarrow$ ADV V_DI VP2 & 0.004367 \\
\hline 7 & VP $\rightarrow$ ADV V_MODAL VP2 & 0.004367 \\
\hline 8 & VP $\rightarrow$ ADV_NEG V_DUOC VP2 & 0.008734 \\
\hline 9 & VP $\rightarrow$ ADV_NEG V_MODAL V VP1 & 0.004367 \\
\hline 10 & VP $\rightarrow$ ADV_NEG V_MODAL V_MODAL VP2 & 0.004367 \\
\hline 11 & $\mathrm{VP} \rightarrow$ ADV_TENSE V VP2 & 0.008734 \\
\hline 12 & $\mathrm{VP} \rightarrow$ ADV_TENSE V_BI VP1 & 0.004367 \\
\hline 13 & $\mathrm{VP} \rightarrow$ ADV_TENSE V_DUOC VP1 & 0.008734 \\
\hline 14 & $\mathrm{VP} \rightarrow$ ADV_TENSE V_DUOC VP2 & 0.004367 \\
\hline 15 & $\mathrm{VP} \rightarrow$ V ADV VP2 & 0.004367 \\
\hline 16 & $\mathrm{VP} \rightarrow$ V ADV_SP VP1 & 0.004367 \\
\hline 17 & $\mathrm{VP} \rightarrow$ V ADV_SP VP2 & 0.004367 \\
\hline 18 & $\mathrm{VP} \rightarrow$ V ADV_SP VP4 & 0.004367 \\
\hline 19 & $\mathrm{VP} \rightarrow$ V V_BI VP2 & 0.004367 \\
\hline 20 & $\mathrm{VP} \rightarrow$ V VP1 & 0.048035 \\
\hline 21 & $\mathrm{VP} \rightarrow$ V VP2 & 0.270742 \\
\hline 22 & $\mathrm{VP} \rightarrow$ V VP3 & 0.026201 \\
\hline 23 & $\mathrm{VP} \rightarrow$ V VP4 & 0.109170 \\
\hline 24 & $\mathrm{VP} \rightarrow$ V V VP2 & 0.008734 \\
\hline 25 & $\mathrm{VP} \rightarrow$ V_BI V VP1 & 0.004367 \\
\hline 26 & $\mathrm{VP} \rightarrow$ V_BI V VP2 & 0.0087367 \\
\hline 27 & $\mathrm{VP} \rightarrow$ V_BI V VP3 & \\
\hline 28 & $\mathrm{VP} \rightarrow$ V_BI V VP4 & 0.004367 \\
\hline & & \\
\hline
\end{tabular}


International Journal on Natural Language Computing (IJNLC) Vol. 2, No.4, August 2013

\begin{tabular}{|l|l|l|}
\hline 29 & VP $\rightarrow$ V_BI VP1 & 0.052402 \\
\hline 30 & $\mathrm{VP} \rightarrow$ V_BI VP2 & 0.034934 \\
\hline 31 & $\mathrm{VP} \rightarrow$ V_BI VP3 & 0.065502 \\
\hline 32 & $\mathrm{VP} \rightarrow$ V_BI VP4 & 0.004367 \\
\hline 33 & $\mathrm{VP} \rightarrow$ V_DI VP2 & 0.026201 \\
\hline 34 & $\mathrm{VP} \rightarrow$ V_DI VP4 & 0.004367 \\
\hline 35 & $\mathrm{VP} \rightarrow$ V_DUOC V VP1 & 0.004367 \\
\hline 36 & $\mathrm{VP} \rightarrow$ V_DUOC V VP2 & 0.017467 \\
\hline 37 & $\mathrm{VP} \rightarrow$ V_DUOC VP1 & 0.004367 \\
\hline 38 & $\mathrm{VP} \rightarrow$ V_DUOC VP2 & 0.008734 \\
\hline 39 & $\mathrm{VP} \rightarrow$ V_DUOC VP3 & 0.004367 \\
\hline 40 & $\mathrm{VP} \rightarrow$ V_MODAL V VP2 & 0.004367 \\
\hline 41 & $\mathrm{VP} \rightarrow$ V_MODAL V_BI VP1 & 0.004367 \\
\hline 42 & $\mathrm{VP} \rightarrow$ V_MODAL V_DUOC VP1 & 0.013100 \\
\hline 43 & $\mathrm{VP} \rightarrow$ V_MODAL VP1 & 0.096070 \\
\hline 44 & $\mathrm{VP} \rightarrow$ V_MODAL VP2 & 0.004367 \\
\hline 45 & $\mathrm{VP} \rightarrow$ V_MODAL VP3 & 0.013100 \\
\hline 46 & $\mathrm{VP} \rightarrow$ V_MODAL VP4 & \\
\hline
\end{tabular}

\subsection{Prepositional phrase $\mathbf{P P}_{-}$}

Table 31. Syntactic rules of $\mathrm{PP}_{-}$

\begin{tabular}{|c|l|c|}
\hline No. & \multicolumn{1}{|c|}{ Rules } & Probabilities \\
\hline 1 & PP_CHO $\rightarrow$ PREP_CHO NP & 1.0 \\
\hline 2 & PP_CUA $\rightarrow$ PREP_CUA NP & 1.0 \\
\hline 3 & PP_DIRECT $\rightarrow$ PREP_DIRECT NP & 1.0 \\
\hline 4 & PP_KHONG $\rightarrow$ PREP_KHONG NP & 1.0 \\
\hline 5 & PP_LOCATION $\rightarrow$ PREP_LOCATION NP & 1.0 \\
\hline 6 & PP_QUA $\rightarrow$ PREP_QUA NP & 1.0 \\
\hline 7 & PP_SAU $\rightarrow$ PREP_SAU NP & 1.0 \\
\hline 8 & PP_TRONG $\rightarrow$ PREP_TRONG NP & 1.0 \\
\hline 9 & PP_TRUOC $\rightarrow$ PREP_TRUOC NP & 1.0 \\
\hline 10 & PP_VAO $\rightarrow$ PREP_VAO NP_TIME & 1.0 \\
\hline 11 & PP_VE $\rightarrow$ PREP_VE NP & 1.0 \\
\hline 12 & PP_VOI $\rightarrow$ PREP_VOI NP & 1.0 \\
\hline
\end{tabular}

\subsection{Adjectival phrase}

Adjectival phrase consists of three types of general adjectival phrase (adjp), adjectival phrase of number (adjp_num), adjectival phrase of number (adjp_measure). 
International Journal on Natural Language Computing (IJNLC) Vol. 2, No.4, August 2013

- General adjectival phrase (ADJP)

Table 32. Syntactic rules of ADJP

\begin{tabular}{|c|l|c|}
\hline No. & \multicolumn{1}{|c|}{ Rules } & Probabilities \\
\hline 1 & ADJP $\rightarrow$ ADJ & 0.797203 \\
\hline 2 & ADJP $\rightarrow$ ADJ ADV_ORDER & 0.055944 \\
\hline 3 & ADJP $\rightarrow$ ADJ ADV_ORDER NP_N & 0.062937 \\
\hline 4 & ADJP $\rightarrow$ ADJ ADV_ORDER NP_NN & 0.020979 \\
\hline 5 & ADJP $\rightarrow$ ADJ ADV_ORDER NP_PROP & 0.006993 \\
\hline 6 & ADJP $\rightarrow$ ADJ ADV_ORDER NP_TIME & 0.006993 \\
\hline 7 & ADJP $\rightarrow$ ADJ ADJ & 0.006993 \\
\hline 8 & ADJP $\rightarrow$ ADJ CONJ ADJ CONJ ADJ & 0.006993 \\
\hline 9 & ADJP $\rightarrow$ ADJ_ORDER & 0.027972 \\
\hline 10 & ADJP $\rightarrow$ SP_WORD ADJ & 0.006993 \\
\hline
\end{tabular}

- Adjectival phrase of number (ADJP_NUM)

Table 33. Syntactic rules of ADJP_NUM

\begin{tabular}{|c|l|c|}
\hline No. & \multicolumn{1}{|c|}{ Rules } & Probabilities \\
\hline 1 & ADJP_NUM $\rightarrow$ ADJ_NUM & 0.759690 \\
\hline 2 & ADJP_NUM $\rightarrow$ ADJ_NUM ADJ_NUM & 0.116279 \\
\hline 3 & ADJP_NUM $\rightarrow$ ADJ_QUANT ADJP_NUM & 0.069767 \\
\hline 4 & ADJP_NUM $\rightarrow$ ADV_EST ADJP_NUM & 0.054264 \\
\hline
\end{tabular}

- Adjectival phrase of measurement (ADJP_MEASURE)

Table 34. Syntactic rules of ADJP_MEASURE

\begin{tabular}{|c|c|c|}
\hline No. & \multicolumn{1}{|c|}{ Rules } & Probabilities \\
\hline 1 & ADJP_MEASURE $\rightarrow$ ADJ_QUANT ADJ_MEASURE & 0.25 \\
\hline 2 & ADJP_MEASURE $\rightarrow$ ADJP_NUM ADJ_MEASURE & 0.75 \\
\hline
\end{tabular}

\section{Syntactic Rules of Sentence}

The probabilities of sentential structure rules are calculated with 1000 Vietnamese training sentences in our TreeBank described in the experiments.

\subsection{Sentences formed by a syntagm}

Table 35. Syntactic rules of sentences formed by a syntagm

\begin{tabular}{|c|c|c|}
\hline No. & \multicolumn{1}{|c|}{ Rules } & Probability \\
\hline 1 & SENTENCE $\rightarrow$ NP & 0.065 \\
\hline 2 & SENTENCE $\rightarrow$ VP2 & 0.006 \\
\hline
\end{tabular}


International Journal on Natural Language Computing (IJNLC) Vol. 2, No.4, August 2013

\begin{tabular}{|c|l|c|}
\hline 3 & SENTENCE $\rightarrow$ VP3 & 0.006 \\
\hline 4 & SENTENCE $\rightarrow$ VP4 & 0.01 \\
\hline 5 & SENTENCE $\rightarrow$ VP & 0.002 \\
\hline
\end{tabular}

\subsection{Sentences formed by two syntagms}

Table 36. Syntactic rules of sentences formed by two syntagms

\begin{tabular}{|c|l|c|}
\hline No. & \multicolumn{1}{|c|}{ Rules } & Probability \\
\hline 1 & SENTENCE $\rightarrow$ NP VP1 & 0.037 \\
\hline 2 & SENTENCE $\rightarrow$ NP VP2 & 0.29 \\
\hline 3 & SENTENCE $\rightarrow$ NP VP3 & 0.156 \\
\hline 4 & SENTENCE $\rightarrow$ NP VP4 & 0.105 \\
\hline 5 & SENTENCE $\rightarrow$ NP VP & 0.178 \\
\hline 6 & SENTENCE $\rightarrow$ VP2 VP2 & 0.003 \\
\hline 7 & SENTENCE $\rightarrow$ VP CONJ VP2 & 0.002 \\
\hline 8 & SENTENCE $\rightarrow$ VP4 VP3 & 0.001 \\
\hline 9 & SENTENCE $\rightarrow$ VP2 PP_LOCATION & 0.007 \\
\hline 10 & SENTENCE $\rightarrow$ VP2 PP_SAU & 0.001 \\
\hline
\end{tabular}

\subsection{Sentences formed by three syntagms}

Table 37. Syntactic rules of sentences formed by three syntagms

\begin{tabular}{|c|l|c|}
\hline No. & \multicolumn{1}{|c|}{ Rules } & Probability \\
\hline 1 & SENTENCE $\rightarrow$ NP VP2 PP_LOCATION & 0.037 \\
\hline 2 & SENTENCE $\rightarrow$ NP VP2 PP_SAU & 0.006 \\
\hline 3 & SENTENCE $\rightarrow$ NP VP2 PP_TRUOC & 0.006 \\
\hline 4 & SENTENCE $\rightarrow$ NP VP2 PP_VOI & 0.003 \\
\hline 5 & SENTENCE $\rightarrow$ NP VP3 PP_LOCATION & 0.011 \\
\hline 6 & SENTENCE $\rightarrow$ NP VP3 PP_VOI & 0.003 \\
\hline 7 & SENTENCE $\rightarrow$ NP VP4 PP_DIRECT & 0.001 \\
\hline 8 & SENTENCE $\rightarrow$ NP VP4 PP_LOCATION & 0.002 \\
\hline 9 & SENTENCE $\rightarrow$ NP VP PP_LOCATION & 0.025 \\
\hline 10 & SENTENCE $\rightarrow$ NP VP PP_SAU & 0.002 \\
\hline 11 & SENTENCE $\rightarrow$ NP VP PP_VAO & 0.001 \\
\hline 12 & SENTENCE $\rightarrow$ NP VP PP_VOI & 0.013 \\
\hline 13 & SENTENCE $\rightarrow$ NP VP2 NP_TIME & 0.003 \\
\hline 14 & SENTENCE $\rightarrow$ NP VP3 NP_TIME & 0.002 \\
\hline 15 & SENTENCE $\rightarrow$ NP VP NP_TIME & 0.003 \\
\hline 16 & SENTENCE $\rightarrow$ NP VP2 VP2 & 0.008 \\
\hline 17 & SENTENCE $\rightarrow$ NP VP2 VP3 & 0.001 \\
\hline 18 & SENTENCE $\rightarrow$ NP VP2 VP & 0.001 \\
\hline 19 & SENTENCE $\rightarrow$ NP VP3 VP2 & 0.001 \\
\hline 20 & SENTENCE $\rightarrow$ NP VP CONJ VP2 & 0.001 \\
\hline 21 & SENTENCE $\rightarrow$ VP2 VP PP_LOCATION & 0.001 \\
\hline
\end{tabular}




\section{EXPERIMENTS}

To train our subcategorized PDCG parser, we build a Treebank with 1000 simple Vietnamese training sentences, which are manually analyzed and tagged by using our Vietnamese subcategorized PDCG grammar and sub-categorical and phrasal tags. All of these Vietnamese sentences are titles of international news (from January 2011 to May 2013) which are collected and selected from the web site of VnExpress [13]. Our Treebank is based on Penn Treebank [14], in which all of the rules are represented by Sandiway Fong's Prolog formats [15].

Based on the built Treebank, the parser can extract 36 sentential rules, 309 phrasal rules and 3248 lexical rules. The probabilities of these rules are calculated by following Probabilistic Definite Clause Grammar [9], [10], [11], and [12].

The results of the experiments of the parser are presented in Table 38. We apply the evaluation method proposed by [16].

Table 38. Results of testing the subcategorized PDCG parser

\begin{tabular}{|c|c|c|c|c|}
\hline Testing datasets & Number of sentences & Precision & Recall & $\mathrm{F}$ \\
\hline Dataset 1 & 250 & 98.46 & 98.50 & 98.48 \\
\hline Dataset 2 & 500 & 98.42 & 98.44 & 98.43 \\
\hline Dataset 3 & 750 & 98.78 & 98.80 & 98.79 \\
\hline Dataset 4 & 1000 & 98.78 & 98.82 & 98.80 \\
\hline
\end{tabular}

For all of experiments, the averages of precisions, recalls and F measures are over $98 \%$.

\section{Conclusions}

The application of Chomsky's principle of subcategorization [8] and PDCG (Probabilistic Definite Clause Grammar) [9], [10], [11], and [12] allows enhancing the precision, recall and $\mathrm{F}$ measures of parsing on all of experimented Vietnamese sentences. However, building a subcategorized PDCG for Vietnamese language requires much time and linguistic complexity in defining tagset, and syntactic rules as well as building a Treebank.

In future works, we prepare to standardize the subcategorization, the tagset and syntactic rules for Vietnamese language. At the same time, the lexicon of parser will be also extended. A strong subcategorized PDCG parser will allow analyzing syntax with better precision.

\section{REFERENCES}

[1] Michael Collins, "Three generative lexicalized models for statistical parsing", Proceeding ACL '98 Proceedings of the 35th Annual Meeting of the Association for Computational Linguistics and Eighth Conference of the European Chapter of the Association for Computational Linguistics, pp. 16-23, 1997.

[2] Michael Collins, "Head-Driven Statisticcal Models for Natural Language Parsing”, Journal Computational Linguistics, MIT Press, Volume. 29, No. 4, pp. 589-637, 2003.

[3] Charniak Eugene, "Statistical techniques for natural language parsing", AI Magazine, 1997. 
International Journal on Natural Language Computing (IJNLC) Vol. 2, No.4, August 2013

[4] Chistopher D. Manning, Hinrich Schütze, Foundations of Statistical Natural Language Processing, MIT Press, 1999.

[5] Nguyen Quoc The, Le Thanh Huong, "Phân tích cú pháp tiếng Việt sử dụng văn phạm phi ngữ cảnh từ vựng hóa kết hợp xác suất”, Proceedings of the FAIR conference, Nha Trang, Vietnam, Aug. 9-10, 2007.

[6] Hoang Anh Viet, Dinh Thi Phuong Thu, Huynh Quyet Thang, "Vietnamese Parsing Applying The PCFG Model", Proceedings of the Second Asia Pacific International Conference on Information Science and Technology, Vietnam, 2007.

[7] Đề tài VLSP. Available at: http://vlsp.vietlp.org:8080 .

[8] Noam Chomsky, Aspects of the theory of syntax, The M.I.T. Press, 1965.

[9] Qaiser Abbas, Nayyara Karamat, Sadia Niazi, "Development of Tree-bank Based Probabilistic Grammar for Urdu Language”, International Journal of Electrical \& Computer Sciences (IJECS), Vol. 9, No. 9, 2009.

[10] Parsing PCFG in Prolog. Available at: http://w3.msi.vxu.se/ nivre/teaching/statnlp/pdcg.html

[11] Assignment for PCFG Parsing. Available at: http://stp.lingfil.uu.se/ nivre/5LN437/statmet_ass2.html

[12] Gerald Gazdar, $1999 . \quad$ Available $\quad$ at: http://www.informatics.sussex.ac.uk/research/groups/nlp/gazdar/teach/nlp/

[13] VnExpress. Available at: http://www.vnexpress.net

[14] Mitchell P. Marcus, Mary Ann Marcinkiewicz, Beatrice Santorini, "Building a Large Annotated Corpus of English: The Penn Treebank", Journal Computational Linguistics - Special issue on using large corpora: II, MIT Press, Volume. 19, No. 2, pp. 313-330, 1993.

[15] Sandiway Fong, Treebank Available http://dingo.sbs.arizona.edu/ sandiway/treebankviewer/index.html

[16] E. Black, "A Procedure for Quantitatively Comparing the Syntactic Coverage of English Grammars", Proceedings DARPA Speech and Natural Language Workshop, Pacific Grove, Morgan Kaufmann, 1991. 